\title{
A VARIATIONAL PRINCIPLE FOR THE HAUSDORFF DIMENSION OF FRACTAL SETS
}

\author{
C. D. CUTLER and L. OLSEN
}

\section{Abstract.}

Let $\mathscr{P}(E)$ denote the set of probability measures on a Borel set $E \subseteq \mathrm{R}^{n}$, and let $\underline{R}(\mu), \bar{R}(\mu)$ denote respectively the lower and upper Rényi dimensions associated with a measure $\mu \in \mathscr{P}(E)$. We prove that the Hausdorff dimension $\operatorname{dim}(E)$ satisfies

$$
\operatorname{dim}(E) \leqq \sup _{\mu \in \mathscr{P}(E)} \underline{R}(\mu)
$$

while, if $E$ is additionally bounded, the packing dimension $\operatorname{Dim}(E)$ satisfies

$$
\operatorname{Dim}(E) \geqq \sup _{\mu \in \mathscr{P}(E)} \bar{R}(\mu) .
$$

As a consequence, for any bounded Borel set $E$ satisfying Taylor's definition of a fractal (i.e. $\operatorname{dim}(E)=\operatorname{Dim}(E))$ we obtain the variational principle

$$
\operatorname{dim}(E)=\operatorname{Dim}(E)=\sup _{\mu \in \mathscr{P}(E)} \underline{R}(\mu)=\sup _{\mu \in \mathcal{P}(E)} \bar{R}(\mu) .
$$

In addition we provide an example showing that the hypothesis "bounded" cannot be eliminated.

\section{Introduction.}

In recent papers on fractals attention has shifted from sets to measure, cf. $[1,2,3,4,5,6,8,9,10,12]$. Thus it seems reasonable to make an attempt at finding a relation between the dimension of a fractal $E$ and parameters connected with measures supported by $E$. Such relations have already been investigated, cf. in particular [14, Theorem 1 p. 62] and Young [18]. Our principal result states that if $E \subseteq \mathrm{R}^{n}$ is a bounded Borel set satisfying Taylor's definition of a fractal, i.e. the Hausdorff dimension $\operatorname{dim}(E)$ of $E$ is equal to the packing dimension $\operatorname{Dim}(E)$ of $E$, cf. [15] and [16], then

$$
\operatorname{dim}(E)=\operatorname{Dim}(E)=\sup _{\mu \in \mathscr{P}(E)} \underline{R}(\mu)=\sup _{\mu \in \mathscr{P}(E)} \bar{R}(\mu)
$$


where $\underline{R}(\mu)$ and $\bar{R}(\mu)$ denote, respectively, the lower and upper Rényi dimensions and $\mathscr{P}(E)$ is the family of all Borel probability measures on $E$.

Formula (1) is a variational principle - i.e. it establishes an equality between a number naturally connected with a space or a map (in this case $\operatorname{dim} E$ ) and the supremum of certain numbers connected to a class of probability measures supported by $E$. It is well-known that variational principles play a major role in ergodic theory (cf. e.g. [17, Chapter 8-9]) since these principles yield a canonical way of choosing measures. Formula (1) yields in a similar way a canonical way of choosing measures - namely measures $\mu \in \mathscr{P}(E)$ such that $\underline{R}(\mu)$ and $\bar{R}(\mu)$ are close to $\operatorname{dim}(E)$ and $\operatorname{Dim}(E)$. It is interesting to note that our variational principle is formulated in terms of the Rényi dimension since generalised Rényi dimensions play an important part in so-called multifractal analysis, cf. e.g. Rand [13] and the references therein.

We begin in section 2 by collecting the relevant facts and setting the notation. Then in section 3 we derive some auxiliary inequalities and prove the variational principle contained in formula (1).

\section{Preliminaries.}

This section contains a survey of the fractal dimensions which we will consider.

Let $(X, d)$ be a separable metric space, $E \subseteq X$ and $s \geqq 0$. Then the s-dimensional Hausdorff measure $\mathscr{H}^{s}(E)$ of $E$ is defined by

$$
\mathscr{H}^{s}(E)=\sup _{\delta>0} \inf \left\{\sum_{i=1}^{\infty}\left(\operatorname{diam} E_{i}\right)^{s} \mid E \subseteq \cup_{i=1}^{\infty} E_{i}, \operatorname{diam} E_{i}<\delta \text { for all } i \in \mathrm{N}\right\} .
$$

The Hausdorff dimension $\operatorname{dim} E$ of $E$ is defined by

$$
\operatorname{dim} E=\inf \left\{s \geqq 0 \mid \mathscr{H}^{s}(E)<\infty\right\}=\sup \left\{s \geqq 0 \mid \mathscr{H}^{s}(E)>0\right\} .
$$

The $s$-dimensional packing measure $\mathscr{P}^{s}(E)$ of $E$ is defined in two stages. First put

$$
\begin{aligned}
\mathscr{P}_{0}^{s}(E) & =\inf _{\delta>0} \sup \left\{\sum_{i=1}^{\infty}\left(\operatorname{diam} B_{i}\right)^{s} \mid B_{i} \cap B_{j}=\varnothing \text { for } i \neq j\right. \\
& \text { and } B_{i} \text { is a closed ball of radius at most } \delta \\
& \text { with center in } E \text { for all } i \in \mathrm{N}\} .
\end{aligned}
$$

Then

$$
\mathscr{P}^{s}(E)=\inf \left\{\sum_{i=1}^{\infty} \mathscr{P}_{0}^{s}\left(E_{i}\right) \mid E \subseteq \cup_{i=1}^{\infty} E_{i}\right\}
$$

The packing dimension $\operatorname{Dim} E$ of $E$ is defined by 


$$
\operatorname{Dim} E=\inf \left\{s \geqq 0 \mid \mathscr{P P}^{s}(E)<\infty\right\}=\sup \left\{s \geqq 0 \mid \mathscr{P P}^{s}(E)>0\right\} .
$$

It is a well-known fact that $\operatorname{dim} E \leqq \operatorname{Dim} E$ for all $E \subseteq \mathrm{R}^{n}$, cf. [14].

Two other useful dimensions of a bounded set $E$ are the upper and lower box dimensions. For each $\delta>0$ let $N_{\delta}(E)$ be the least number of sets of diameter at most $\delta$ that cover $E$. Then the upper and lower box dimensions of $E$ are defined by

$$
\bar{C}(E)=\limsup _{\delta \rightarrow 0} \frac{\log N_{\delta}(E)}{-\log \delta}
$$

and

$$
\underline{C}(E)=\liminf _{\delta \rightarrow 0} \frac{\log N_{\delta}(E)}{-\log \delta}
$$

respectively.

Let us introduce the Rényi dimension. Fix $\mu \in \mathscr{P}(X)$ and write

$$
\begin{gathered}
h_{r}(\mu)=\inf \left\{-\sum_{i=1}^{\infty} \mu\left(E_{i}\right) \log \mu\left(E_{i}\right) \mid\left(E_{i}\right)_{i}\right. \text { is a countable Borel } \\
\text { partition of } \left.X \text { and diam } E_{i} \leqq r\right\}
\end{gathered}
$$

for $r>0$. Then the upper and lower Rényi dimensions of $\mu$ are defined by

$$
\bar{R}(\mu)=\limsup _{r \rightarrow 0}-\frac{h_{r}(\mu)}{\log r}
$$

and

$$
\underline{R}(\mu)=\liminf _{r \rightarrow 0}-\frac{h_{r}(\mu)}{\log r}
$$

respectively, (cf. [18]).

\section{Inequalities and the Variational Principle.}

We want to prove that

$$
\operatorname{dim}(E) \leqq \sup _{\mu \in \mathscr{P}(E)} \underline{R}(\mu)
$$

for a Borel subset $E$ of $\mathrm{R}^{n}$, and

$$
\operatorname{Dim}(E) \geqq \sup _{\mu \in \mathscr{P}(E)} \bar{R}(\mu)
$$

for a bounded Borel subset $E$ of $\mathrm{R}^{n}$. Both proofs are based on the following result: 
THEOREM 1. Let $E \subseteq \mathrm{R}^{n}$ be a Borel set. Then the following assertions hold:

i)

$$
\operatorname{dim}(E)=\sup _{\mu \in \mathscr{P}(E)}\left(\inf _{x \in E} \liminf \underset{r \downarrow 0}{ } \frac{\log \mu(B(x, r))}{\log r}\right)
$$

ii) If

$$
E \subseteq\left\{x \mid \limsup _{r \downarrow 0} \frac{\log \mu(B(x, r))}{\log r} \geqq \alpha\right\} \text { and } \mu(E)>0 \text {, }
$$

then

$$
\operatorname{Dim}(E) \geqq \alpha .
$$

ProOF. i) Follows easily from [14, Theorem 1]. ii) Follows from [14, Theorem 1], however see also Theorem 3.2 of [5].

We begin with three small technical lemmas

LEMMA 2. Let $\mu$ be a Borel probability measure on $\mathrm{R}^{n}$. Let $E$ be a Borel set, $t \geqq 0$ and $\delta \in] 0,1[$. Suppose

$$
\log \mu(B(x, r)) \leqq t \log r
$$

for all $x \in E$ and $r \in] 0, \delta[$. Then

$$
\underline{R}(\mu) \geqq \mu(E) t .
$$

PRoOF. Let $r \in] 0, \delta\left[\right.$ and $\left(E_{i}\right)_{i}$ be a partition of $\mathrm{R}^{n}$ such that diam $\left(E_{i}\right) \leqq r$. Let $I=\left\{i \mid E_{i} \cap E \neq \varnothing\right\}$. If $i \in I$ then we can choose a point $x_{i} \in E_{i} \cap E$ such that $E_{i} \subseteq B\left(x_{i}, r\right)$, whence

$$
\log \mu\left(E_{i}\right) \leqq \log \mu\left(B\left(x_{i}, r\right)\right) \leqq t \log r \text { for } i \in I .
$$

By (4) we have

$$
\begin{aligned}
-\sum_{i} \mu\left(E_{i}\right) \log \mu\left(E_{i}\right) & \geqq-\sum_{i \in I} \mu\left(E_{i}\right) \log \mu\left(E_{i}\right) \geqq-\sum_{i \in I} \mu\left(E_{i}\right) t \log r \\
& =-\mu\left(\bigcup_{i \in I} E_{i}\right) t \log r \geqq-\mu(E) t \log r .
\end{aligned}
$$

Since the partition $\left(E_{i}\right)_{i}$ was arbitrary this inequality implies that

$$
\left.h_{r}(\mu) \geqq-\mu(E) t \log r \text { for } r \in\right] 0, \delta[
$$

whence

$$
\underline{R}(\mu)=\liminf _{r \rightarrow 0}-\frac{h_{r}(\mu)}{\log r} \geqq t \mu(E) .
$$


LEMMA 3. Let $F \subseteq \mathrm{R}^{n}$ be a bounded Borel set and $r>0$. Then there exists a finite collection $F_{1}, \ldots, F_{m}$ of disjoint Borel sets with diam $\left(F_{i}\right) \leqq r$ such that $F \subseteq \cup_{i} F_{i}$ and such that for each $i$, there exists an $x_{i} \in F$ satisfying

$$
B\left(x_{i}, \frac{1}{4} r\right) \subseteq F_{i} \text {. }
$$

Proof. Construct a sequence of balls $B\left(x_{1}, \frac{1}{2} r\right), B\left(x_{2}, \frac{1}{2} r\right), \ldots$ such that $x_{i} \in F$ and $d\left(x_{i}, x_{j}\right)>\frac{1}{2} r$ for $i \neq j$. Because $F$ is totally bounded this process must terminate at some finite stage, giving balls $B\left(x_{1}, \frac{1}{2} r\right), \ldots, B\left(x_{m}, \frac{1}{2} r\right)$ such that any $x \in F$ must satisfy $\min _{i} d\left(x, x_{i}\right) \leqq \frac{1}{2} r$ (consequently $F \subseteq \cup_{i=1}^{m} B\left(x_{i}, \frac{1}{2} r\right)$ ). Note that the smaller balls $B\left(x_{1}, \frac{1}{4} r\right), \ldots, B\left(x_{m}, \frac{1}{4} r\right)$ are disjoint. Set

$$
\begin{aligned}
& F_{1}=B\left(x_{1}, \frac{1}{2} r\right) \backslash \bigcup_{j=2}^{m} B\left(x_{j}, \frac{1}{4} r\right) \\
& F_{i}=B\left(x_{i}, \frac{1}{2} r\right) \backslash\left(\bigcup_{j=1}^{i-1} F_{j} \cup \bigcup_{j=i+1}^{m} B\left(x_{j}, \frac{1}{4} r\right)\right) \text { for } i=2, \ldots, m-1 \\
& F_{m}=B\left(x_{m}, \frac{1}{2} r\right) \backslash \bigcup_{j=1}^{m-1} F_{i} .
\end{aligned}
$$

It is clear that the $F_{i}$ 's are disjoint, and since $B\left(x_{1}, \frac{1}{4} r\right), \ldots, B\left(x_{m}, \frac{1}{4} r\right)$ are disjoint we can conclude that $B\left(x_{i}, \frac{1}{4} r\right) \subseteq F_{i}$ and $F \subseteq \cup_{i} F_{i}$.

LEMma 4. Let $E \subseteq \mathrm{R}^{n}$ be a bounded Borel set and $\mu \in \mathscr{P}(E)$. Let $F \subseteq E$ be a Borel set, $t \geqq 0$ and $\delta \in] 0,1[$. Assume

$$
\log \mu(B(x, r)) \geqq t \log r
$$

for all $x \in F$ and $0<r<\delta$. Then

$$
\bar{R}(\mu) \leqq t+\mu(E \backslash F) \bar{C}(E \backslash F) .
$$

Proof. Let $r \in] 0, \delta$ [ and choose by Lemma 3 a finite pairwise disjoint cover$\operatorname{ing}\left(F_{1}, \ldots, F_{m}\right)$ of $F$ with diam $F_{i} \leqq r$ and such that there exists points $x_{i} \in F$ for all $i$ satisfying

$$
B\left(x_{i}, \frac{1}{4} r\right) \subseteq F_{i} .
$$

The set $E \backslash F$ can be covered by $N=N_{r}(E \backslash F)$ closed balls $B_{1}, \ldots, B_{N}$ of diameter at most $r$. Define $Q_{1}, \ldots, Q_{N}$ by

$$
\begin{aligned}
& Q_{1}=\left(B_{1} \cap(E \backslash F)\right) \backslash \cup_{j} F_{j} \\
& Q_{i}=\left(B_{i} \cap(E \backslash F)\right) \backslash\left(\cup_{j} F_{j} \cup \cup_{j=1}^{i-1} Q_{j}\right) \text { for } i=2, \ldots, N .
\end{aligned}
$$

Then $F_{1}, \ldots, F_{m}, Q_{1}, \ldots, Q_{N}$ are disjoint sets of diameter not exceeding $r$, and

$$
E=\cup_{i}\left(F_{i} \cap E\right) \cup \cup_{i} Q_{i}, \quad \cup_{i} Q_{i} \subseteq E \backslash F .
$$


Hence

$$
\begin{aligned}
h_{r}(\mu) & \leqq-\sum_{i=1}^{m} \mu\left(F_{i} \cap E\right) \log \mu\left(F_{i} \cap E\right)-\sum_{i=1}^{N} \mu\left(Q_{i}\right) \log \mu\left(Q_{i}\right) \\
& =-\sum_{i=1}^{m} \mu\left(F_{i}\right) \log \mu\left(F_{i}\right)-\sum_{i=1}^{N} \mu\left(Q_{i}\right) \log \mu\left(Q_{i}\right) \\
& \leqq-\sum_{i=1}^{m} \mu\left(F_{i}\right) \log \mu\left(B\left(x_{i}, \frac{1}{4} r\right)\right)-\sum_{i=1}^{N} \mu\left(Q_{i}\right) \log \mu\left(Q_{i}\right) \\
& \leqq-\sum_{i=1}^{m} \mu\left(F_{i}\right) t \log \left(\frac{1}{4} r\right)-\sum_{i=1}^{N} \mu\left(Q_{i}\right) \log \mu\left(Q_{i}\right) \\
& \leqq-t \log \left(\frac{1}{4} r\right)-\sum_{i=1}^{N} \mu\left(Q_{i}\right) \log \mu\left(Q_{i}\right) .
\end{aligned}
$$

We know that if $p_{1}, \ldots, p_{k} \geqq 0$ and $\sum_{i=1}^{k} p_{i}=s \in[0,1]$ then in fact $-\sum_{i=1}^{k} p_{i}$ $\log p_{i} \leqq s \log k-s \log s \leqq s \log k+\frac{1}{e}$. Therefore

$$
\begin{aligned}
h_{r}(\mu) & \leqq-t \log \left(\frac{1}{4} r\right)+\sum_{i=1}^{N} \mu\left(Q_{i}\right) \log N+\frac{1}{e} \\
& \leqq-t \log \left(\frac{1}{4} r\right)+\mu\left(\bigcup_{i=1}^{N} Q_{i}\right) \log N_{r}(E \backslash F)+\frac{1}{e} \\
& \leqq-t \log \left(\frac{1}{4} r\right)+\mu(E \backslash F) \log N_{r}(E \backslash F)+\frac{1}{e}
\end{aligned}
$$

for $r<\delta$, whence

$$
\begin{aligned}
\bar{R}(\mu)=\limsup _{r \downarrow 0} \frac{h_{r}(\mu)}{-\log r} & \leqq \limsup _{r \downarrow 0}\left(\frac{t \log \left(\frac{1}{4} r\right)}{\log r}+\mu(E \backslash F) \frac{\log N_{r}(E \backslash F)}{-\log r}-\frac{1}{e \log r}\right) \\
& \leqq t+\mu(E \backslash F) \bar{C}(E \backslash F) .
\end{aligned}
$$

We are now ready to prove (2) and (3).

Proposition 5. Let $E \subseteq \mathrm{R}^{n}$. Then the following assertions hold:

i) If $E$ is a Borel set then

$$
\operatorname{dim} E \leqq \sup _{\mu \in \mathscr{P}(E)} \underline{R}(\mu) .
$$

ii) If $E$ is a bounded Borel set then 


$$
\sup _{\mu \in \mathscr{P}(E)} \bar{R}(\mu) \leqq \operatorname{Dim} E .
$$

Proof. i) Let $t<\operatorname{dim} E$. Then Theorem 1 part i) implies that there exists a measure $\mu \in \mathscr{P}(E)$ such that

$$
t<\liminf _{r \downarrow 0} \frac{\log \mu(\mathrm{B}(\mathrm{x}, r))}{\log r} \text { for all } x \in E .
$$

Now put

$$
E_{m}=\left\{x \in E \mid \frac{\log \mu(B(x, r))}{\log r}>t \text { for } 0<r<\frac{1}{m}\right\}, m \in N .
$$

Let $\varepsilon>0$ and observe that (5) implies that $E_{m} \uparrow E$. We can thus choose an integer $N \in \mathrm{N}$ so $\mu\left(E_{N}\right) \geqq \mu(E)-\varepsilon=1-\varepsilon$. An application of Lemma 2 then yields

$$
\sup _{\lambda \in \mathscr{P}(E)} \underline{R}(\lambda) \geqq \underline{R}(\mu) \geqq \mu\left(E_{N}\right) t \geqq(1-\varepsilon) t
$$

which proves the first part of the proposition since $t<\operatorname{dim} E$ and $\varepsilon>0$ were arbitrary.

ii) Let $\mu \in \mathscr{P}(E)$ and $t>\operatorname{Dim}(E)$. Then Theorem 1 part ii) implies that

$$
\limsup _{r \downarrow 0} \frac{\log \mu(B(x, r))}{\log r} \leqq \operatorname{Dim}(E) \mu \text {-a.s. }
$$

and we can thus choose a subset $F$ of $E$ with $\mu(F)=1$ such that lim sup $r \downarrow 0$ $\frac{\log \mu(B(x, r))}{\log r}<t$ for all $x \in F$. Now put

$$
F_{m}=\left\{x \in F \mid \frac{\log \mu(B(x, r))}{\log r}<t \text { for } 0<r<\frac{1}{m}\right\}, m \in N .
$$

An application of Lemma 4 then yields

$$
\bar{R}(\mu) \leqq t+\mu\left(E \backslash F_{m}\right) \bar{C}(E)=t+\mu\left(F \backslash F_{m}\right) \bar{C}(E) .
$$

Since $F_{m} \uparrow F$ we conclude that $\bar{R}(\mu) \leqq t$. This completes the proof since both $\mu \in \mathscr{P}(E)$ and $t>\operatorname{Dim}(E)$ were arbitrary.

Proposition 5 immediately yields the following variational principle

Proposition 6. If $E \subseteq \mathrm{R}^{n}$ is a bounded Borel set satisfying $\operatorname{dim}(E)=\operatorname{Dim}(E)$, then

$$
\operatorname{dim}(E)=\operatorname{Dim}(E)=\sup _{\mu \in \mathscr{P}(E)} \underline{R}(\mu)=\sup _{\mu \in \mathscr{P}(E)} \bar{R}(\mu)
$$


It is easily seen that the inequality in Proposition 5) ii) may not hold if the assumption "bounded" is omitted. Indeed put $E=N$ and $q_{n}=$ $c\left((n+1)(\log (n+1))^{2}\right)^{-1}$ for $n \in N$ where $c=1 / \sum_{n=2}^{\infty} \frac{1}{n(\log n)^{2}}$, and define $\mu \in \mathscr{P}(E)$ by $\mu=\sum_{n} q_{n} \delta_{n}$ (here $\delta_{x}$ denotes the Dirac measure concentrated at $x$ ). If $0<r<1$ and $\left(E_{i}\right)_{i}$ is a countable partition of $E=N$ then $\left(E_{i} \cap E\right)_{i}=(\{n\})_{n \in N}$, whence

$$
\frac{h_{r}(\mu)}{-\log r}=\frac{-\sum_{n} \mu(\{n\}) \log (\mu\{n\})}{-\log r}=\frac{-\sum_{n} q_{n} \log q_{n}}{-\log r}=\infty
$$

which implies that $\bar{R}(\mu)=\underline{R}(\mu)=\infty>0=\operatorname{Dim}(E)$.

\section{REFERENCES}

1. M. Barnsley, Fractals Everywhere, Academic Press, 1988.

2. M. Barnsley, S. Demko, J. Elton and J. Geronimo, Invariant measures for Markov processes arising from iterated function systems with place-dependent probabilitites, Ann. Inst. $\mathrm{H}$. Poincaré Prob. Statist. 24 (1988), 367-394.

3. M. Barnsley and J. Elton, A new class of Markov processes for image encoding, J. of Appl. Prob. Statist. 20 (1988), 14-32.

4. C. D. Cutler, Connecting ergodicity and dimension in dynamical systems, Ergodic Theory Dynamical Systems 10 (1990), 451-462.

5. C. D. Cutler, Measure disintegrations with respect to $\sigma$-stable monotone indices and the pointwise representation of packing dimension, Proceedings of the Measure Theory Conference at Oberwolfach. In Supplemento ai Rendiconti del Circolo Matematico di Palermo, Ser. II, No. 28, 1992, 319-339.

6. J. Elton \& Z. Yan, Approximation of Measures by Markov Processes and Homogeneous Affine Iterated Function System, Constr. Approx. 5 (1989), 69-88.

7. K. Falconer, Fractal Geometry-Mathematical Foundations and Applications, John Wiley \& Sons, 1990.

8. J. Geronimo \& D. Hardin, An exact formula for the measure dimensions associated with a class of piecewise linear maps, Const. Approx. 5 (1989), 89-98.

9. H. Haase, On the dimension of product measures, Mathematika 37 (1990), 316-323.

10. H. Haase, Dimension of measures, Acta Univ. Carolin. Math. Phys. 31 (1990), 29-34.

11. J. Hutchinson, Fractals and self-similarity, Indiana Univ. Math. J. 30 (1981), 713-747.

12. J.-P. Kahane and Y. Katznelson, Décomposition des mesures selon la dimension, Colloquium Mathematicum 63 (1990), 269-279.

13. D. A. Rand, The singularity spectrum $f(\alpha)$ for cookie-cutters, Ergodic Theory Dynamical Systems 9 (1989), 527-541.

14. C. Tricot, Two definitions of fractional dimension, Math. Proc. Camb. Philos. Soc. 91 (1982), $57-74$.

15. S. J. Taylor, The measure theory of random fractals, Math. Proc. Cambridge Philos Soc. 100 (1986), 383-406.

16. S. J. Taylor, A measure theory definition of fractals, Proceedings of the Measure Theory Conference at Oberwolfach. In Supplemento ai Rendiconti del Circolo Matematico di Palermo, Ser. II, No. 28, 1992, 371-378. 
17. P. Walters, An Introduction to Ergodic Theory, Springer-Verlag, 1982.

18. L-S. Young, Dimension, entropy and Lyapunov Exponents, Ergodic Theory Dynamical Systems 2 (1982), 109-124.

DEPARTMENT OF STATISTICS AND ACTUARIAL SCIENCE UNIVERSITY OF WATERLOO

WATERLOO, ONTARIO

CANADA N2L 3G1

\author{
MATEMATISK INSTITUT \\ UNIVERSITY OF COPENHAGEN \\ 2100 KØBENHAVN $\emptyset$ \\ DENMARK
}

